\title{
Resonant interaction of Rossby waves in two-dimensional flow on a plane
}

AUTHOR(S):

Yamada, Michio; Yoneda, Tsuyoshi

\section{CITATION:}

Yamada, Michio ...[et al]. Resonant interaction of Rossby waves in twodimensional flow on a plane. Physica D: Nonlinear Phenomena 2013, 245(1): 1-7

\section{ISSUE DATE:}

2013-02

URL:

http://hdl.handle.net/2433/168839

\section{RIGHT:}

(C) 2012 Elsevier B.V.; This is not the published version. Please cite only the published version.; この論文は出版社版でありません。引用の際に は出版社版をご確認ご利用ください。 


\title{
Resonant interaction of Rossby waves in two-dimensional flow on a $\beta$ plane
}

\author{
Michio Yamada \\ Research Institute for Mathematical Sciences, Kyoto University, Kyoto, 606-8502, \\ Japan \\ Tsuyoshi Yoneda \\ Department of Mathematics, Hokkaido University, Sapporo 060-0810, Japan
}

\begin{abstract}
An incompressible two-dimensional flow on a $\beta$ plane is considered. Rossby waves are generally expected to dominate the $\beta$ plane dynamics, and here in this paper we prove a mathematically rigorous theorem that at a high $\beta$, the flow dynamics are governed exclusively by the resonant interactions of Rossby waves.
\end{abstract}

Key words: Navier-Stokes equations, $\beta$ plane, Rossby wave, mild solution

\section{Introduction}

We consider three-wave interactions of the Rossby waves in an incompressible two-dimensional flow on a $\beta$ plane, governed by the following equation,

$$
\partial_{t} \zeta+J(\Psi, \zeta)+\beta \partial_{x} \Psi=\nu \Delta \zeta
$$

where $\zeta=\zeta(t, x)\left(t>0, x=\left(x_{1}, x_{2}\right) \in \mathbb{R}^{2}\right), J(A, B)=\left(\partial_{x_{1}} A\right)\left(\partial_{x_{2}} B\right)-$ $\left(\partial_{x_{1}} B\right)\left(\partial_{x_{2}} A\right) . \Psi$ is the streamfunction of the fluid with the fluid velocity being given by $u=\left(u_{1}, u_{2}\right)=\left(-\partial_{x_{2}} \Phi, \partial_{x_{1}} \Phi\right)$, and $\zeta=\Delta \Psi$ is the vorticity. The initial condition is $\zeta(0, x)=\zeta_{0}(x)$.

Email addresses: yamada@kurims.kyoto-u.ac.jp (Michio Yamada), yoneda@math.sci.hokudai.ac.jp (Tsuyoshi Yoneda). 
The $\beta$-plane approximation was first introduced by meteorologists (see $[3,4])$ as a tangent plane of a sphere to approximately describe fluid motion on a rotating sphere, assuming that the Colioris parameter is a linear function of the latitude. Conventionally the $x_{1}$ - and $x_{2}$-axes are respectively taken eastward and northward, and the $x_{1}$-direction is often called zonal direction in earth and planetary sciences. We employ this intuitive terminology in this paper when convenient. A formal derivation of the $\beta$-plane approximation is given in [11]. The equation (1) describes a two-dimensional motion of an incompressible fluid on the $\beta$ plane, and it has been known that in its solution, as time goes on, a zonal pattern, consisting of alternating eastward and westward zonal flows, similar to the zonal band structure observed on Jupiter, ${ }^{1}$ emerges.

The equation (1) has been widely employed in earth and planetary sciences to study the effect of differential rotation (latitudinal variation of the Coriolis parameter) and the mechanism of zonal flow formation. From a physical point of view, one of the most important properties of equation (1), in contrast with non-rotating two-dimensional Navier-Stokes fluid, is that it permits linear waves called Rossby waves. The Rossby wave solution, originates from the third term of (1), with the dispersion relation,

$$
\omega=-\frac{\beta k_{1}}{k_{1}^{2}+k_{2}^{2}}
$$

where $\omega$ and $\left(k_{1}, k_{2}\right)$ are the angular frequency and the wavenumber vector. The Rossby wave is known to be an important origin of characteristic behaviors of atmosphere and ocean.

Generally in nonlinear dynamics of linear waves, resonant pairs are expected to give a significant contribution to the nonlinear interactions. Also in the case of the Rossby waves, the resonant waves have been considered to play important roles in the dynamics of geophysical fluids $[10,13]$. Here in this paper, we prove a mathematical theorem which supports the importance of the resonant pairs of Rossby waves. We consider the time evolution of a flow field which is governed by (1), but with the nonlinear terms being restricted only to the resonant pairs of Rossby waves. Our theorem asserts that the flow field approximates the original flow field as precisely as desired by taking a large $\beta$. The theorem means that under a high rotation rate, the time evolution of the flow field is governed by the resonant interaction of the Rossby waves. For pure mathematical analysis of the rotating Navier-Stokes equations, from which some of mathematical techniques are employed in this paper, refer to $[1,2,5-9,15]$.

This paper is organized as follows: In section 2, we show the existence of

\footnotetext{
1 The origin of the zonal band structure on Jupiter is still controversial. Three
} dimensional deep convection is another possible origin of the surface zonal bands. 
local-in-time unique solution to (1), where the existence time is independent of the parameter $\beta$. In sections 3 , we give a resonant-nonresonant decomposition, and state the main theorem. The decomposition is discussed in detail in Section 4. In the last section, we complete the proof of the main theorem.

\section{Local existence and uniqueness of the solution}

In physical discussions it is almost always assumed that a unique and smooth solution to (1) exists for $0 \leq t<\infty$ for an appropriate initial condition. In this section, however, we would like to give a proof for the local-in-time existence of the solution for the purpose of logical ensuring that our discussion is not meaningless. Readers who are not interested in the local existence and uniqueness theorem can skip most of this section.

We will discuss our problem on $\mathbb{T}^{2}$, i.e. $[0,2 \pi] \times[0,2 \pi]$ with the periodic boundary conditions with period $2 \pi$ in both $x_{1}$ - and $x_{2}$ - directions. We expand the vorticity $\zeta$ into Fourier series which will be employed in the discussions below. We introduce a space $X^{s}(s \geq 0)$ of the vorticity functions by using a weighted $\ell^{1}$ space of Fourier coefficients as

$$
\begin{aligned}
& X^{s}:=\left\{\zeta=\sum_{n \in \mathbb{Z}^{2}} a_{n} e^{i n \cdot x} \in \mathcal{S}^{\prime}\left(\mathbb{T}^{2}\right)\right. \\
& \text { for } \quad a=\left\{a_{n}\right\}_{n \in \mathbb{Z}^{2}}: a_{n}=a_{-n}^{*}\left(n \in \mathbb{Z}^{2}\right), \\
& \left.\|a\|_{s}:=\|a\|_{\ell_{1}^{s}}:=\sum_{n \in \mathbb{Z}^{2}}\left(1+|n|^{2}\right)^{s / 2}\left|a_{n}\right|<\infty\right\} .
\end{aligned}
$$

Here $\mathcal{S}^{\prime}\left(\mathbb{T}^{2}\right)$ is the space of the tempered distributions on $\mathbb{T}^{2}$ and $a_{n}^{*}$ is the complex conjugate of $a_{n}$. It is well known that $X^{0}$ is an algebra which is continuously embedded in $B U C$, the space of bounded uniformly continuous functions. Under the periodic boudary condition, we can point out the following relationship between $X^{s}\left(\mathbb{T}^{2}\right)$ and the Hölder space $C^{s}\left(\mathbb{T}^{2}\right)$.

Proposition 1 We have $\zeta \in C^{\infty}\left(\mathbb{T}^{2}\right)$ if and only if $\zeta \in \cap_{s \geq 1} X^{s}$. Obviously, $X^{s} \subset C^{s}(s \geq 0)$.

Throughout in this paper, we measure the magnitude of the Fourier coefficients $a$ mostly by $\ell^{1}$-norm, and we often write $\|a\|_{0}$ as $\|a\|$. To treat the Coriolis term $\beta \partial_{x} \Psi$ in (1), we need to define the multipliers $n_{j} /|n|^{2}(j=1,2)$ for $n=\left(n_{1}, n_{2}\right) \in \mathbb{Z}^{2}$ as

$$
\lim _{\epsilon \rightarrow 0} \frac{n_{j}}{|n|^{2}+\epsilon}
$$


which gives

$$
\left.\frac{n_{j}}{|n|^{2}}\right|_{n=0}=0
$$

It is clear, by rescaling the quantities, that we can assume $\nu=1$ without loss of generality.

Expanding the initial condition and the vorticity into Fourier series as

$$
\begin{aligned}
\zeta_{0}(x) & :=\sum_{n \in \mathbb{Z}^{2}} a_{0, n} e^{i n \cdot x}, \\
\zeta(t, x) & :=\sum_{n \in \mathbb{Z}^{2}} a_{n}(t) e^{i n \cdot x},
\end{aligned}
$$

we rewrite the evolution equation (1) into the equation in $\ell_{1}\left(\mathbb{Z}^{2}\right)$ for Fourier coefficients as follow,

$$
\begin{aligned}
a_{n}(0) & =a_{0, n}, \\
\partial_{t} a_{n}(t)+|n|^{2} a_{n}(t) & +\beta \omega(n) a_{n}(t) \\
& =\sum_{n=k+m}\left(\frac{k_{1} m_{2}}{|k|^{2}}-\frac{k_{2} m_{1}}{|k|^{2}}\right) a_{k}(t) a_{m}(t) \\
& =\sum_{n=k+m}\left(\frac{k_{1} n_{2}}{|k|^{2}}-\frac{k_{2} n_{1}}{|k|^{2}}\right) a_{k}(t) a_{m}(t) \\
& =: J_{n}(a, a)
\end{aligned}
$$

for $n \in \mathbb{Z}^{2}$. for $n \in \mathbb{Z}^{2}$ where $\omega(n):=i n_{1} /|n|^{2}$.

We now state a local existence theorem in $\ell_{1}\left(\mathbb{Z}^{2}\right)$ as follows:

Theorem 2 Assume that $a(0):=\left\{a_{n}(0)\right\}_{n \in \mathbb{Z}^{2}} \in \ell_{1}\left(\mathbb{Z}^{2}\right)$. Then there is a local existence time $T_{L}$ and a local-in-time unique solution $a(t):=\left\{a_{n}(t)\right\}_{n \in \mathbb{Z}^{2}} \in$ $C\left(\left[0, T_{L}\right]: \ell_{1}\left(\mathbb{Z}^{2}\right)\right)$ satisfying

$$
T_{L} \geq \frac{C}{\left\|a_{0}\right\|^{2}}, \quad \sup _{0<t<T_{L}}\|a(t)\| \leq 2\left\|a_{0}\right\|
$$

where $C$ is a positive constant independent of $\beta$. Moreover if $\|a(0)\|_{s}<\infty$ for $s \geq 0$, then we have the following pointwise estimate:

$$
\left|a_{n}(t)\right| \leq \frac{C_{1}}{\left(1+|n|^{2}\right)^{s / 2}} \quad \text { for } \quad 0<t<T_{L}
$$

where $C_{1}>0$ is independent of $\beta$. 
Proof. The solution $a(t)$ satisfies the integral equation,

$a_{n}(t)=e^{-\left(|n|^{2}+\beta \omega(n)\right) t} a_{n}(0)+\int_{0}^{t} e^{-\left(|n|^{2}+\beta \omega(n)\right)(t-\tau)} J_{n}(a(\tau), a(\tau)) d \tau, \quad\left(n \in \mathbb{Z}^{2}\right)$.

A direct calculation shows that

$$
\left|a_{n}(t)\right| \leq\left|a_{n}(0)\right|+\int_{0}^{t} \frac{C}{(t-\tau)^{1 / 2}}\left|J_{n}(a(\tau), a(\tau))\right| d \tau, \quad\left(n \in \mathbb{Z}^{2}\right),
$$

where we have used the following inequality for $\tau=1$,

$$
\sup _{t>0, n \in \mathbb{Z}^{2}} t^{\tau / 2}|n|^{\tau} e^{-|n|^{2} t} \leq C \text { for } s>0 .
$$

Evaluation of the convolution in the Jacobian leads to the estimate:

$$
\|a(t)\| \leq\|a(0)\|+C t^{1 / 2}\left(\sup _{0<\tau \leq t}\|a(\tau)\|\right)^{2} \quad \text { for } \quad t>0,
$$

We obtain the local existence result from the above estimate (see [6] for example). More precisely, let us define $\left\{h^{j}(t)\right\}_{j \in \mathbb{N}}=\left\{h_{n}^{j}(t)\right\}_{j \in \mathbb{N}, n \in \mathbb{Z}^{2}}$ inductively as

$$
\begin{aligned}
h_{n}^{0}(t) & =e^{-\left(|n|^{2}+\beta \omega(n)\right) t} a_{n}(0), \\
h_{n}^{j+1}(t) & =h_{n}^{0}(t)+\int_{0}^{t} e^{-\left(|n|^{2}+\beta \omega(n)\right)(t-\tau)} J_{n}\left(h^{j}(\tau), h^{j}(\tau)\right) d \tau .
\end{aligned}
$$

First we show $\left\{h^{j}(t)\right\}_{j \in \mathbb{N}}$ is uniformly bounded with respect to $j \geq 1$, i.e. if $h^{0} \in Y$ and $h^{j} \in Y$, then $h^{j+1} \in Y$, where

$$
Y:=\left\{h \in C\left([0, T]: \ell_{1}\left(\mathbb{Z}^{2}\right)\right): \sup _{0<t \leq T}\|h(t)\| \leq 2\|a(0)\|\right\},
$$

in which $T$ will be determined later. A direct calculation leads to

$$
\left\|h^{j+1}(t)\right\| \leq\|a(0)\|+T^{1 / 2}\left(\sup _{0<\tau \leq T}\left\|h^{j}(\tau)\right\|\right)^{2} \quad \text { for } \quad 0<t \leq T .
$$

which yields

$$
\left\|h^{j+1}(t)\right\| \leq\|a(0)\|+T^{1 / 2} 4\|a(0)\|^{2} \quad \text { for } \quad 0<t \leq T .
$$

Setting $T=1 /\left(16\|a(0)\|^{2}\right)$, we have

$$
\sup _{0<t \leq T}\left\|h^{j+1}(t)\right\| \leq 2\|a(0)\|
$$


which means that $h^{j+1} \in Y$.

Next we show that $\left\{h^{j}\right\}_{j}$ is a Cauchy sequence, namely, $\sup _{0<t \leq T} \| h^{j}(t)-$ $h^{k}(t) \|$ tends to zero for $j, k \rightarrow \infty(j \leq k)$. This means the existence of the local-in-time solution since the function space $C\left([0, T]: \ell_{1}\left(\mathbb{Z}^{2}\right)\right)$ is complete.

Because $J_{n}\left(h^{j}, h^{j}\right)-J_{n}\left(h^{j-1}, h^{j-1}\right)=J_{n}\left(h^{j}-h^{j-1}, h^{j}\right)-J_{n}\left(h^{j-1}, h^{j-1}-h^{j}\right)$, we have from $(7)$ and $(8)$

$\left\|h^{j+1}(t)-h^{j}(t)\right\| \leq C t^{1 / 2} \sup _{0<\tau \leq t}\left\|h^{j}(\tau)-h^{j-1}(\tau)\right\|\left(\sup _{0<\tau \leq t}\left\|h^{j}(\tau)\right\|+\sup _{0<\tau \leq t}\left\|h^{j-1}(\tau)\right\|\right)$.

and thus

$$
\left\|h^{j+1}(t)-h^{j}(t)\right\| \leq 4 T\|a(0)\| \sup _{0<\tau \leq T}\left\|h^{j}(\tau)-h^{j-1}(\tau)\right\| \quad \text { for } \quad 0<t \leq T .
$$

by the use fo the uniform boundedness of $h^{j}(\tau)$. Since $T=1 /(16\|a(0)\|)$, we finally have

$$
\left\|h^{j+1}(t)-h^{j}(t)\right\| \leq \frac{1}{4} \sup _{0<\tau \leq T}\left\|h^{j}(\tau)-h^{j-1}(\tau)\right\| \quad \text { for } \quad 0<t \leq T .
$$

which shows that $\left\{h^{j}(t)\right\}_{j}$ is a Cauchy sequence in $C\left([0, T]: \ell_{1}\left(\mathbb{Z}^{2}\right)\right)$. Therefore $h^{j}(t)$ converges to a solution of (5). The uniqueness also follows from the contraction (9).

In order to obtain the pointwise bound (4), it suffices to show that if $\|a(0)\|_{s} \leq C$ where $C$ is independent of $\beta$, then there is some $T_{L}(>0)$ such that

$$
\|a(t)\|_{s} \leq C \text { for } t \in\left[0, T_{L}\right],
$$

uniformly for $s \geq 0$, because then (10) and the estimate,

$$
\sup _{n}\left\{\left(1+|n|^{2}\right)^{s / 2}\left|a_{n}(t)\right|\right\} \leq\|a(t)\|_{s}, \quad\left(0<t<T_{L}\right)
$$

gives the pointwise bound (4). To show (10), we use a bootstrapping argument. Namely, we first control $\sup _{0<t \leq T_{L}}\|a(t)\|_{1 / 2}$ by using the integral equation 5 . Then we control subsequently $\sup _{t}\|a(t)\|_{1}, \sup _{0<t \leq T_{L}}\|a(t)\|_{3 / 2}$ and so on. For the case of $s=1 / 2$, we have from (6),

$$
\|a(t)\|_{1 / 2} \leq\left\|a_{n}(0)\right\|_{1 / 2}+C t^{1 / 4} \sup _{0<\tau \leq t}\|a(\tau)\|^{2} \quad \text { for } \quad 0<t<T_{L} .
$$

which shows that $\sup _{0<t \leq T_{L}}\|a(t)\|_{1 / 2}$ is controllable. A similar calculation gives the disired estimates for $\|a(t)\|_{1},\|a(t)\|_{3 / 2}, \cdots$, in sequence. 


\section{Resonant-nonresonant decomposition and the main theorem}

In this section, we give a resonant-nonresonant decomposition and a statement of the main theorem. We define $c_{n}(t):=e^{-\beta \omega(n) t} a_{n}(t)$ for $n \in \mathbb{Z}^{2}$, which satisfy the following equation,

$$
\begin{aligned}
& c_{n}(0)=a_{n}(0), \\
& \partial_{t} c_{n}(t)=-|n|^{2} c_{n}(t)+B_{n}(c(t), c(t)),
\end{aligned}
$$

where

$$
B_{n}\left(c_{1}, c_{2}\right):=e^{-\beta \omega(n) t} J_{n}\left(e^{t \beta \omega(\cdot)} c_{1}, e^{t \beta \omega(\cdot)} c_{2}\right) .
$$

We decompose the nonlinear operator $B_{n}$ into two parts, the resonant part $B_{n}^{0}(\cdot, \cdot)$ which is independent of $\beta$, and the nonresonant part $B_{n}^{0+}(\beta t, \cdot, \cdot)$ which depends on $\beta$ (The details are given in Section 4 ). The former part $B_{n}^{0}$ consists of the nonlinear interactions of $c_{k}$ and $c_{m}$ which satisfy the resonance condition

$$
n=k+m, \quad \text { and } \quad \omega(n)=\omega(k)+\omega(m),
$$

while the latter part $B_{n}^{0+}$ consists of $c_{k}$ and $c_{m}$ which does not satisfy the resonance condition. The resonant part $B_{n}^{0}$ depends on time only implicitly through $c_{k}$ and $c_{n}$, while the nonresonant part $B_{n}^{0+}$ does explicitly through $\beta t$. Therefore we have

$$
\begin{aligned}
& c_{n}(0)=a_{n}(0), \\
& \partial_{t} c_{n}(t)=-|n|^{2} c_{n}(t)+B_{n}^{0}(c(t), c(t))+B_{n}^{0+}(\beta t, c(t), c(t)) .
\end{aligned}
$$

We will give a detailed discussion on these parts in the next section.

Now we define the solution $b(t)$ to the resonant part of the equation as

$$
\begin{aligned}
& b_{n}(0)=c_{n}(0)=a_{n}(0), \\
& \partial_{t} b_{n}(t)=-|n|^{2} b_{n}(t)+B_{n}^{0}(b(t), b(t))
\end{aligned}
$$

which we call the limit equation. The remainder term $r_{n}(t):=c_{n}(t)-b_{n}(t)$ then obeys the following equation,

$$
\partial_{t} r_{n}(t)=-|n|^{2} r_{n}(t)+B_{n}^{0}(c(t), r(t))+B_{n}^{0}(r(t), b(t))+B_{n}^{0+}(\beta t, c(t), c(t))
$$

with $r_{n}(0)=0$. Intuitively, the limit equation corresponds to the case of $\beta \rightarrow \infty$ in (1) as the time dependence of $B_{n}^{0+}$ is then so fast that the net contribution of $B_{n}^{0+}$ is expected to disappear. This kind of decomposition 
has already been done for the rotating 3D-Navier-Stokes/Euler equations (see $[1,2,9,15]$ for example). It is known by numerical integration that for large values of $\beta$, the flow field is non-isotropic as there arise zonal flows in $x_{1}$ direction, which means that a significant part of the energy is concentrated to the Fourier components of $n \sim\left(0, n_{2}\right)$, suggesting that the solution to the limit equation is also non-isotropic.

The main result of this paper is now stated: the solution $c(t)$ to (12) tends to the solution $b(t)$ to the limit equation provided that $\beta$ tends to infinity. More precisely,

Theorem 3 For all $\epsilon>0$, there is $\beta_{0}>0$ s.t. $\|r(t)\| \leq \epsilon$ for $0<t<T_{L}$ and $|\beta|>\beta_{0}$, where $T_{L}$ is the local existence time (see Theorem 2).

The proof is given in the following sections.

\section{Detailed decomposition of the nonlinear term}

We have decomposed the nonlinear term into the resonant part and the nonresonant part. In this section we further decompose the resonant part and estimate the resonant and the nonresonant terms.

Theorem 4 Let $c_{1}(t):=\left\{c_{1, n}(t)\right\}_{n \in \mathbb{Z}^{2}}$ and $c_{2}(t):=\left\{c_{2, n}(t)\right\}_{n \in \mathbb{Z}^{2}}$ are series in $\ell^{1}\left(\mathbb{Z}^{2}\right)$. Also let $\omega_{n k m}:=(1 / i)(\omega(n)-\omega(k)-\omega(m))=n_{1} /|n|^{2}-k_{1} /|k|^{2}-$ $m_{1} /|m|^{2}$, and

$$
b_{n k m}\left(c_{k}, c_{m}\right):=\left(\frac{k_{1} m_{2}}{|k|^{2}}-\frac{k_{2} m_{1}}{|k|^{2}}\right) c_{k}(t) c_{m}(t)=\left(\frac{k_{1} n_{2}}{|k|^{2}}-\frac{k_{2} n_{1}}{|k|^{2}}\right) c_{k}(t) c_{m}(t),
$$

where $n=k+m$.

Then the nonlinear term

$$
B_{n}\left(c_{1}, c_{2}\right):=e^{-\beta \omega(n) t} J_{n}\left(e^{t \beta \omega(\cdot)} c_{1}, e^{\beta \omega(\cdot) t} c_{2}\right)=\sum_{n=k+m} e^{i \beta \omega_{n k m} t} b_{n k m}\left(c_{1, k}, c_{2, m}\right)
$$

are decomposed into the resonant part,

$$
B_{n}^{0}\left(c_{1}, c_{2}\right)=\sum_{\substack{n=k+m \\ \omega_{n k m}=0}} b_{n k m}\left(c_{1, k}, c_{2, m}\right)
$$


and the nonresonant part

$$
B_{n}^{0+}\left(\beta t, c_{1}, c_{2}\right):=\sum_{\substack{n=k+m \\ \omega_{n k m} \neq 0}} e^{i \beta \omega_{n k m} t} b_{n k m}\left(c_{1, k}, c_{2, m}\right) .
$$

The resonant part is further decomposed as

$$
B_{n}^{0}\left(c_{1}, c_{2}\right):=\sum_{\mu \in D} B_{n}^{\mu}\left(c_{1}, c_{2}\right)
$$

for $D=\{(0,0,0),(0,1,1),(1,0,1),(1,1,0),(1,1,1)\}$, where the bilinear forms $B^{\mu}:=\left\{B_{n}^{\mu}\right\}_{n \in \mathbb{Z}^{2}}(\mu \in D)$ are defined as follows:

$$
\begin{aligned}
& B_{n}^{(0,0,0)}\left(c_{1}, c_{2}\right):= \sum_{\substack{n=k+m, \omega_{n k m}=0 \\
n_{1}=k_{1}=m_{1}=0}} b_{n k m}\left(c_{1, k}, c_{2, m}\right), \\
& B_{n}^{(0,1,1)}\left(c_{1}, c_{2}\right):= \sum_{\substack{n=k+m_{n k m}=0 \\
-k_{1}=m_{n} \neq 0}} b_{n k m}\left(c_{1, k}, c_{2, m}\right), \\
& B_{n}^{(1,0,1)}\left(c_{1}, c_{2}\right):= \sum_{\substack{n=k+m, \omega_{n k m}=0 \\
n_{1}=m_{1} \neq 0}} b_{n k m}\left(c_{1, k}, c_{2, m}\right), \\
& B_{n}^{(1,1,0)}\left(c_{1}, c_{2}\right):= \sum_{\substack{n=k+m, \omega_{n k m}=0 \\
n_{1}=k_{1} \neq 0}} b_{n k m}\left(c_{1, k}, c_{2, m}\right), \\
& B_{n}^{(1,1,1)}\left(c_{1}, c_{2}\right):=\sum_{\substack{n=k+m, \omega_{n k m}=0 \\
n_{1}, k_{1}, m_{1} \neq 0}} b_{n k m}\left(c_{1, k}, c_{2, m}\right) .
\end{aligned}
$$

Moreover the resonant and the nonresonant parts have the following estimation,

$$
\begin{aligned}
\left\|\left\{e^{-|n|^{2} t} B_{n}\left(c_{1}, c_{2}\right)\right\}_{n \in \mathbb{Z}^{2}}\right\| & \leq\left(C / t^{1 / 2}\right)\left\|c_{1}\right\|\left\|c_{2}\right\| \\
\left\|\left\{e^{-|n|^{2} t} B_{n}^{0+}\left(\beta t, c_{1}, c_{2}\right)\right\}_{n \in \mathbb{Z}^{2}}\right\| & \leq\left(C / t^{1 / 2}\right)\left\|c_{1}\right\|\left\|c_{2}\right\|,
\end{aligned}
$$

where $C(>0)$ is independent of $\beta$.

We note that $\mu=\left(\mu_{1}, \mu_{2}, \mu_{3}\right)$ shows whether each of the $x_{1}$-components of the wavenumbers $n, k, m$ vanishes or not; for example, if $\mu=(1,1,0)$, then $n_{1}=k_{1} \neq 0$ and $m_{1}=0$. Also note that from the inequalities (22) and (23), we can show the local existence for $b$ and $r$. The proof is quite similar to that of Theorem 2, and thus we omit its detail.

For the description of resonant interaction, let us introduce a wavenumber set of the Fourier coefficients $b=\left\{b_{n}\right\}_{n \in \mathbb{Z}^{2}} \in \ell_{1}\left(\mathbb{Z}^{2}\right)$ as 


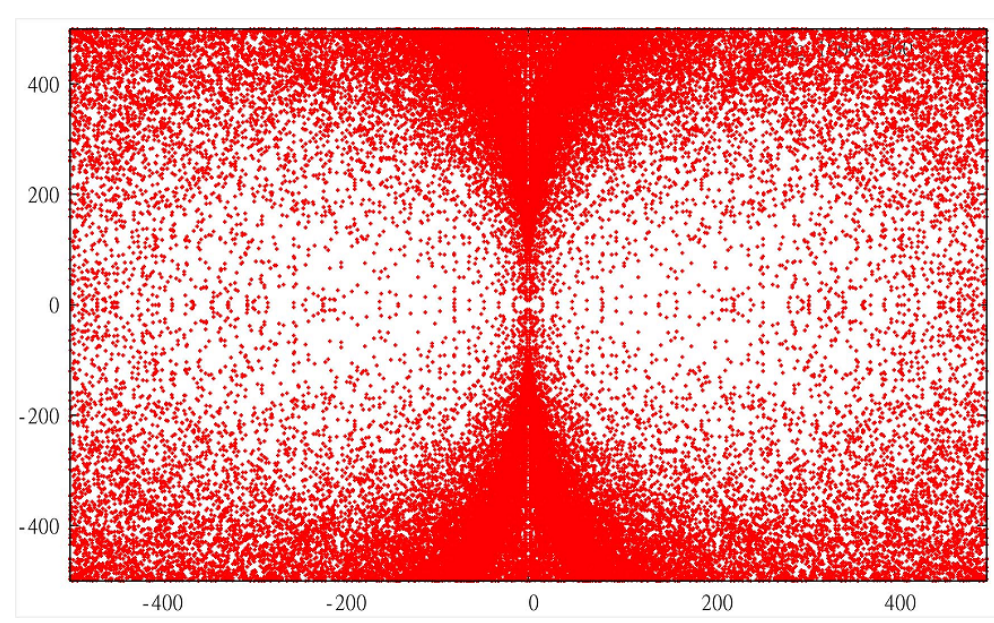

Fig. 1. The wavenumber set $\Lambda$ in $-500<n_{1}, n_{2}<500$. The horizontal and vertical axes are $n_{1}$ and $n_{2}$, respectively. We see that $\Lambda$ is quite an anisotropic set.

$$
\begin{aligned}
\Lambda^{b}:= & \left\{n \in \mathbb{Z}^{2}: b_{n_{1}, n_{2}} \neq 0\right\} \cup\left\{n \in \mathbb{Z}^{2}: b_{-n_{1}, n_{2}} \neq 0\right\} \\
& \cup\left\{n \in \mathbb{Z}^{2}: b_{n_{1},-n_{2}} \neq 0\right\} \cup\left\{n \in \mathbb{Z}^{2}: b_{-n_{1},-n_{2}} \neq 0\right\},
\end{aligned}
$$

and define a trivial resonance as a resonance between the resonant wavenumber pairs $n, k, m$ with $n_{1} k_{1} m_{1}=0$. The following proposition is then immediately obtained.

Proposition 5 (Trivial resonance) For any $b_{1}, b_{2} \in \ell_{1}\left(\mathbb{Z}^{2}\right)$, we have

$$
\begin{aligned}
& \Lambda^{B^{(1,1,0)}\left(b_{1}, b_{2}\right)} \subset \Lambda^{b_{1}}, \quad \Lambda^{B^{(1,0,1)}\left(b_{1}, b_{2}\right)} \subset \Lambda^{b_{2}}, \\
& \Lambda^{B^{(0,0,0)}\left(b_{1}, b_{2}\right)} \subset \Lambda_{0} \quad \text { and } \quad \Lambda^{B^{(0,1,1)}\left(b_{1}, b_{2}\right)} \subset \Lambda_{0} .
\end{aligned}
$$

where $\Lambda_{0}=\left\{n \in \mathbb{Z}^{2}: n_{1}=0\right\}$.

Note that $B_{n}^{(1,1,0)}\left(b_{1}, b_{2}\right)$ is not zero only for $n$ which satisfies the resonance condition. The only possible pair is a pair of trivial resonance, $n=\left(n_{1}, n_{2}\right), k=$ $\left(n_{1},-n_{2}\right), m=\left(0,2 n_{2}\right)$, which implies $\Lambda^{B^{(1,1,0)}\left(b_{1}, b_{2}\right)} \subset \Lambda^{b_{1}}$. The rest cases are similarly shown.

Let us define another wavenumber set consisting of wavenumbers in nontrivial resonance.

Definition 6 (Wavenumber set of non-trivial resonance) Let $\Lambda$ be a wavenumber set such that $n \in \mathbb{Z}^{2}$ if and only if there is a wavenumber pair of non-trivial resonance including $n$.

The red points in Figure 1 shows wavenumber set $\Lambda$. It should be remarked that the wavenumber set $\Lambda$ is quite anisotropic with more wavenumbers being along the $x_{2}$-axis, suggesting that the flow field arising from within $\Lambda$ is nearly zonal. Note that the flow field arising from within $\Lambda_{0}$ is purely zonal. 
It is easily seen that if $\Lambda^{b} \subset \Lambda$, then $\Lambda^{B^{(1,1,1)}(b, b)} \subset \Lambda$. Therefore, if $\Lambda^{b} \subset$ $\Lambda \cup \Lambda_{0}$, then the resonant interactions $B^{(1,0,1)}(b, b), B^{(1,1,0)}(b, b), B^{(1,1,1)}(b, b)$ vanish at wavenumbers outside $\Lambda \cup \Lambda_{0}$, while $B^{(0,0,0)}(b, b)$ and $B^{(0,1,1)}(b, b)$ vanish outside $\Lambda_{0}$. In other words, if the initial Fourier coefficients $c(0)$ satisfies $\Lambda^{c(0)} \subset \Lambda \cup \Lambda_{0}$, and if only the resonant nonlinear interactions are taken into account, then $\Lambda^{c(t)} \subset \Lambda \cup \Lambda_{0}$ for $0 \leq t<T_{L}$.

Here we should make a physical comment. Generally in a turbulent solution of the governing equation (1), every wavenumber component has nonzero energy. Suppose that the initial energy distribution in wavenumber space is isotropic. Two-dimensional turbulence is known to transfer the energy from small- to large-scale motions (energy inverse cascade). If there is no effect of rotation, i.e. $\beta=0$, then the energy therefore becomes concentrated isotropically around the origin in wavenumber space. However, when $\beta \neq 0$, the energy transfer becomes governed by the resonant interaction of Rossby waves, and the number of the resonant triads gives a rough estimate of the strength of the nonlinear energy transfer. Therefore, roughly speaking, the wavenumbers in the white area in Figure 1 are then expected to gain less energy compared with wavenumbers in the red area, consistently with the observed non-isotropic zonal structure in the flow field.

Proof of Theorem 4. We define a set of resonant wavenumber pairs, $\mathcal{K} \subset$ $\left(\mathbb{Z}^{2}\right)^{3}$, and supplimentary sets $\left(\mathbb{Z}^{2}\right)_{\mu}^{3}$, as

$$
\mathcal{K}:=\left\{(n, k, m) \in\left(\mathbb{Z}^{2}\right)^{3}: \omega_{n k m}=0\right\}
$$

and

$$
\left(\mathbb{Z}^{2}\right)_{\mu}^{3}:=\left\{(n, k, m) \in\left(\mathbb{Z}^{2}\right)^{3}: n_{1} \in \mathbb{Z}_{\mu_{1}}, k_{1} \in \mathbb{Z}_{\mu_{2}} \quad \text { and } \quad m_{1} \in \mathbb{Z}_{\mu_{3}}\right\}
$$

for $\mu=\left(\mu_{1}, \mu_{2}, \mu_{3}\right) \in\{0,1\}^{3}$, where $\mathbb{Z}_{0}:=\{0\}$ and $\mathbb{Z}_{1}:=\mathbb{Z} \backslash\{0\}$. The set of nonresonant wavenumber pairs is defined as $\mathcal{K}^{c}=\left(\mathbb{Z}^{2}\right)^{3} \backslash \mathcal{K}$. Then we have a decomposition of $\left(\mathbb{Z}^{2}\right)^{3}$,

$$
\left(\mathbb{Z}^{2}\right)^{3}=\mathcal{K} \cup \mathcal{K}^{c}=\bigcup_{\mu \in\{0,1\}^{3}}\left(\mathcal{K} \cap\left(\mathbb{Z}^{2}\right)_{\mu}^{3}\right) \cup \mathcal{K}^{c}
$$

where

$$
\left(\mathcal{K} \cap\left(\mathbb{Z}^{2}\right)_{\mu}^{3}\right) \cap\left(\mathcal{K} \cap\left(\mathbb{Z}^{2}\right)_{\mu^{\prime}}^{3}\right)=\emptyset \quad \text { if } \quad \mu \neq \mu^{\prime}
$$

With $\mathcal{K}$ and $\left(\mathbb{Z}^{2}\right)_{\mu}^{3}$, we can express the bilinear forms $B_{n}^{\mu}, B_{n}^{0+}$ as 


$$
\begin{gathered}
B_{n}^{\mu}\left(c_{1}, c_{2}\right):=\sum_{\substack{n=k+m \\
(n, k, m) \in \mathcal{K} \cap\left(\mathbb{Z}^{2}\right)_{\mu}^{3}}} b_{n k m}\left(c_{1, k}, c_{2, m}\right) \quad \text { (resonant part), } \\
B_{n}^{0+}\left(\beta t, c_{1}, c_{2}\right):=\sum_{\substack{n=k+m \\
(n, k, m) \in \mathcal{K}^{c}}} e^{i \beta \omega_{n k m} t} b_{n k m}\left(c_{1, k}, c_{2, m}\right) \quad \text { (nonresonant part) }
\end{gathered}
$$

for $\mu \in\{0,1\}^{3}$. We have immediately estimates of these bilinear forms:

$$
\left|B_{n}^{\mu}\left(c_{1}, c_{2}\right)\right| \leq \sum_{n=k+m}|n|\left|c_{1, k}\right|\left|c_{2, m}\right|
$$

and

$$
\left|B_{n}^{0+}\left(\beta t, c_{1}, c_{2}\right)\right| \leq \sum_{n=k+m}|n|\left|c_{1, k}\right|\left|c_{2, m}\right|
$$

which give (22) and (23) by the use of (6). A straightforward calculation yields

$$
B_{n}(c(t), c(t))=\sum_{\mu \in\{0,1\}^{3}} B_{n}^{\mu}(c(t), c(t))+B_{n}^{0+}(\beta t, c(t), c(t)),
$$

and lastly, to show (16), it suffices to have the following three equalities:

$$
B_{n}^{(1,0,0)}(c, c)=0, B_{n}^{(0,1,0)}(c, c)=0, B_{n}^{(0,0,1)}(c, c)=0 \quad \text { for } \quad c=\left\{c_{n}\right\}_{n \in \mathbb{Z}^{2}}
$$

which are easily obtained because, for example, if $k_{1}=m_{1}=0$ then $B_{n}^{(1,0,0)}$ vanishes from (3). The other two equalities are similarly shown, and the proof is completed.

\section{Proof of the main theorem}

In this section we give a proof of the main result, Theorem 3, based on the results in previous sections. The point of the proof is to control the oscillatory integral of the nonresonant part, where the frequency mismatch $\omega_{n k m}$ is in its denominator and can take an arbitrary small value. We avoid this point by dividing the integral into two parts and estimate them separately.

Proof of Theorem 3. First let us define an oscillatory integral of the nonresonant part as follows:

$$
\tilde{B}_{n}^{0+}\left(\beta t, c_{1}, c_{2}\right):=\sum_{\substack{n=k+m \\ \omega_{n k m} \neq 0}} \frac{1}{i \beta \omega_{n k m}} e^{i \beta \omega_{n k m} t} b_{n k m}\left(c_{1, k}, c_{2, m}\right),
$$


where

$$
b_{n k m}\left(c_{k}, c_{m}\right):=\left(\frac{k_{1} m_{2}}{|k|^{2}}-\frac{k_{2} m_{1}}{|k|^{2}}\right) c_{k}(t) c_{m}(t)=\left(\frac{k_{1} n_{2}}{|k|^{2}}-\frac{k_{2} n_{1}}{|k|^{2}}\right) c_{k}(t) c_{m}(t) .
$$

We note that

$$
\begin{aligned}
& \partial_{t}\left(\tilde{B}_{n}^{0+}\left(\beta t, c_{1}(t), c_{2}(t)\right)\right)= \\
& \quad B_{n}^{0+}\left(\beta t, c_{1}(t), c_{2}(t)\right)+\tilde{B}_{n}^{0+}\left(\beta t, \partial_{t} c_{1}(t), c_{2}(t)\right)+\tilde{B}_{n}^{0+}\left(\beta t, c_{1}(t), \partial_{t} c_{2}(t)\right)
\end{aligned}
$$

and, from (23),

$$
\left\|\left\{e^{-|n|^{2} t} \tilde{B}_{n}^{0+}\left(\beta t, c_{1}, c_{2}\right)\right\}_{n \in \mathbb{Z}^{2}}\right\| \leq \frac{C}{t^{1 / 2} \tau \beta}\left\|c_{1}\right\|\left\|c_{2}\right\|,
$$

where $\tau$ is the infimum of $\left\{\left|\omega_{n k m}\right|\right\}$ over all combinations of $n, k$ and $m$ with $n=k+m$. The oscillatory integral $\tilde{B}_{n}^{0+}$ would allow us to control the remainder term since $\beta$ is in the denominator. However $\omega_{n k m}$ is also in the denominator and there is a subsequence of $\left\{\left|\omega_{n k m}\right|\right\}$ which converges to 0 , meaning that we cannot control $\left\|\left\{e^{-|n|^{2} t} \tilde{B}_{n}^{0+}\left(\beta t, c_{1}, c_{2}\right)\right\}_{n \in \mathbb{Z}^{2}}\right\|$ directly. Therefore in order to keep $\tau$ apart from zero, we need to handle only finite elements of $b(t)=$ $\left\{b_{n}(t)\right\}_{n \in \mathbb{Z}^{2}}, c(t)=\left\{c_{n}(t)\right\}_{n \in \mathbb{Z}^{2}}$ and $r(t)=\left\{r_{n}(t)\right\}_{n \in \mathbb{Z}^{2}}$. Below we first give a formal calculation, and after that we make it rigorous.

Let $\tilde{B}^{0+}:=\left\{\tilde{B}_{n}^{0+}\right\}_{n}$. For the time being, we assume that $\tilde{B}^{0+}$ have only finite elements, and explain the outline of the proof. In order to estimate the remainder term $r(t)=\left\{r_{n}(t)\right\}_{n \in \mathbb{Z}^{2}}$, it suffices to estimate $y_{n}(t):=$ $r_{n}(t)-\tilde{B}_{n}^{0+}(\beta t, c(t), c(t))$, since $\tilde{B}^{0+}$ tends to zero in $\ell^{1}$-norm when $\beta \rightarrow \infty$ (if $\tilde{B}^{0+}$ have only finite elements). We see from (15) and (27) that the functions $\left\{y_{n}(t)\right\}_{n}$ satisfy the following equations:

$$
\partial_{t} y_{n}(t)+|n|^{2} y_{n}(t)-L_{n}(c(t), b(t), y(t))=\sum_{j=1}^{3} E_{n}^{j}
$$

where

$$
\begin{aligned}
L_{n}(c, b, y) & :=B_{n}^{0}(c, y)+B_{n}^{0}(y, b), \\
E_{n}^{1} & :=-\tilde{B}_{n}^{0+}\left(\beta t, \partial_{t} c(t), c(t)\right)-\tilde{B}_{n}^{0+}\left(\beta t, c(t), \partial_{t} c(t)\right), \\
E_{n}^{2} & :=-|n|^{2} \tilde{B}_{n}^{0+}(\beta t, c(t), c(t)), \\
E_{n}^{3} & :=L_{n}\left(c(t), b(t), \tilde{B}^{0+}(\beta t, c, c)\right) .
\end{aligned}
$$

Note that we can also estimate $\left\{\partial_{t} c_{n}(t)\right\}_{n}$ and $\left\{E_{n}^{2}\right\}_{n}$ in $\ell^{1}$-norm if they have only finite elements. Using (28) together with the estimate of the resonant 
part (22), (23) and Gronwall's inequality (for the detail, see the next rigorous calculation), we can control the remainder term.

Now we give a rigorous discussion with more detailed computation. To control $r(t)$, we split it into two parts: finitely many terms $\mathcal{P}_{\eta} r$ and small (in $\left.\ell^{1}\left(\mathbb{Z}^{2}\right)\right)$ remainder terms $\left(I-\mathcal{P}_{\eta}\right) r($ cf. [1, Theorem 6.3] and [15]), where we have defined the projection operator,

$$
\mathcal{P}_{\eta} r:=\left\{r_{n}:|n| \leq \eta\right\} .
$$

Note that $\mathcal{P}_{\eta} r$ and $\left(I-\mathcal{P}_{\eta}\right) r$ correspond respectively to a lower and a higher wavenumber parts, satisfying $\left\|\left(I-\mathcal{P}_{\eta}\right) r\right\| \rightarrow 0 \quad(\eta \rightarrow \infty)$.

It should be remarked that we have the following estimates:

$$
\begin{aligned}
\left\|\mathcal{P}_{\eta} \tilde{B}^{0+}\left(\beta t, \mathcal{P}_{\eta} c_{1}, \mathcal{P}_{\eta} c_{2}\right)\right\| & \leq \frac{\alpha(\eta)}{\beta}\left(1+\eta^{2}\right)^{1 / 2}\left\|\mathcal{P}_{\eta} c_{1}\right\|\left\|\mathcal{P}_{\eta} c_{2}\right\|, \\
\left\|\mathcal{P}_{\eta} B^{0+}\left(\beta t, \mathcal{P}_{\eta} c_{1}, \mathcal{P}_{\eta} c_{2}\right)\right\| & \leq\left(1+\eta^{2}\right)^{1 / 2}\left\|\mathcal{P}_{\eta} c_{1}\right\|\left\|\mathcal{P}_{\eta} c_{2}\right\| \\
\left\|\mathcal{P}_{\eta}\left(|\cdot|^{2} y\right)\right\| & \leq\left(1+\eta^{2}\right)\left\|\mathcal{P}_{\eta} y\right\| \\
\left\|\partial_{t} \mathcal{P}_{\eta} c\right\| & \leq\left\|\mathcal{P}_{\eta}\left(|\cdot|^{2} c\right)\right\|+\left\|\mathcal{P}_{\eta} B(c, c)\right\| \\
& \leq\left(1+\eta^{2}\right)\|c\|+\left(1+\eta^{2}\right)^{1 / 2}\|c\|^{2}
\end{aligned}
$$

for $0<t<T_{L}$, where $|\cdot| y$ means a series $\left\{|n|^{2} y_{n}\right\}_{n \in \mathbb{Z}^{2}}$, and

$$
\alpha(\eta):=\max \left\{\left|\omega_{n k m}\right|^{-1}:|n|,|k|,|m| \leq \eta, \quad n=k+m\right\} .
$$

Note that $\alpha(\eta)$ is always finite, since we now have only a finite number of nonresonant combinations of $n, k$ and $m$.

We set $\tilde{y}_{n}:=r_{n}-\tilde{B}_{n}^{0+}\left(\beta t, \mathcal{P}_{\eta} c, \mathcal{P}_{\eta} c\right)$ and $\tilde{L}_{n}(c, b, \tilde{y}):=B_{n}^{0}(c, \tilde{y})+B_{n}^{0}(\tilde{y}, b)$. Note that $\tilde{y}_{n}(0)=-\tilde{B}_{n}^{0+}\left(0, \mathcal{P}_{\eta} c(0), \mathcal{P}_{\eta} c(0)\right)$, and it still have $\beta$ in the denominator. For $|n| \leq \eta$, we see that

$$
\begin{aligned}
\partial_{t}\left(\tilde{y}_{n}+\tilde{B}_{n}^{0+}\right)= & -|n|^{2}\left(\tilde{y}_{n}+\tilde{B}_{n}^{0+}\right)+\tilde{L}_{n}\left(c, b, \mathcal{P}_{\eta}\left(\tilde{y}+\tilde{B}^{0+}\right)\right) \\
& +\tilde{L}_{n}\left(c, b,\left(I-\mathcal{P}_{\eta}\right)\left(\tilde{y}+\tilde{B}^{0+}\right)\right) \\
& +B_{n}^{0+}\left(\beta t, \mathcal{P}_{\eta} c, \mathcal{P}_{\eta} c\right)+B_{n}^{0+}\left(\beta t,\left(I-\mathcal{P}_{\eta}\right) c, \mathcal{P}_{\eta} c\right) \\
& +B_{n}^{0+}\left(\beta t, c,\left(I-\mathcal{P}_{\eta}\right) c\right),
\end{aligned}
$$

and we obtain from $(27)$,

$$
\partial_{t} \tilde{y}_{n}+|n|^{2} \tilde{y}_{n}-\tilde{L}_{n}\left(c, b, \mathcal{P}_{\eta} \tilde{y}\right)=\sum_{j=1}^{3} \tilde{E}_{n}^{j}+R_{n}
$$


for $|n| \leq \eta$, where

$$
\begin{aligned}
& \tilde{E}_{n}^{1}:=-\tilde{B}_{n}^{0+}\left(\beta t, \partial_{t} \mathcal{P}_{\eta} c, \mathcal{P}_{\eta} c\right)-\tilde{B}_{n}^{0+}\left(\beta t, \mathcal{P}_{\eta} c, \partial_{t} \mathcal{P}_{\eta} c\right), \\
& \tilde{E}_{n}^{2}:=-|n|^{2} \tilde{B}_{n}^{0+}\left(\beta t, \mathcal{P}_{\eta} c, \mathcal{P}_{\eta} c\right), \\
& \tilde{E}_{n}^{3}:=\tilde{L}_{n}\left(c, b, \mathcal{P}_{\eta} \tilde{B}_{n}^{0+}\left(\beta t, \mathcal{P}_{\eta} c, \mathcal{P}_{\eta} c\right)\right) \text {, } \\
& R_{n}:=\tilde{L}_{n}\left(c, b,\left(I-\mathcal{P}_{\eta}\right)\left(\tilde{y}+\tilde{B}^{0+}\right)\right) \\
& +B_{n}^{0+}\left(\beta t,\left(I-\mathcal{P}_{\eta}\right) c, \mathcal{P}_{\eta} c\right)+B_{n}^{0+}\left(\beta t, c,\left(I-\mathcal{P}_{\eta}\right) c\right) .
\end{aligned}
$$

Equation (31) is a linear heat type equation with external forces $\tilde{E}^{1}, \tilde{E}^{2}$, $\tilde{E}^{3}$ and $R$. We see that for any $\epsilon>0$, there is $\eta_{0}$ such that if $\eta>\eta_{0}$, then $\left\|\mathcal{P}_{\eta} R\right\|<\epsilon$ because $\left\|\left(1-\mathcal{P}_{\eta}\right) a\right\| \rightarrow 0$ as $\eta \rightarrow \infty\left(a \in \ell_{1}\left(\mathbb{Z}^{1}\right)\right)$. The pointwise bound (10) permits us to choose $\eta_{0}$ independently of $\beta$. We can also see from (30) that for any $\epsilon>0$, there is $\beta_{0}$ (depending on $\eta_{0}$ ) such that if $|\beta|>\beta_{0}$, then $\sum_{j=1}^{3}\left\|\mathcal{P}_{\eta} \tilde{E}^{j}\right\|<\epsilon$. Thus we obtain from the integral equation (5) and (30), the estimate

$$
\begin{aligned}
\left\|\mathcal{P}_{\eta} \tilde{y}(t)\right\| \leq & \frac{\alpha(\eta)}{\beta}\left(1+\eta^{2}\right)^{1 / 2}\|c(0)\|^{2}+ \\
& \int_{0}^{t} \frac{C}{(t-\tau)^{1 / 2}}\left((\|c(\tau)\|+\|b(\tau)\|)\left\|\mathcal{P}_{\eta} \tilde{y}(\tau)\right\|+\epsilon\right) d \tau \\
\leq & \frac{\alpha(\eta)}{\beta}\left(1+\eta^{2}\right)^{1 / 2}\|a(0)\|^{2}+ \\
& \int_{0}^{t} \frac{C}{(t-\tau)^{1 / 2}}\left(4\|a(0)\|\left\|\mathcal{P}_{\eta} \tilde{y}(\tau)\right\|+\epsilon\right) d \tau
\end{aligned}
$$

More precisely, let us take $t_{0}(<t)$ in order to satisfy

$$
\int_{0}^{t_{0}} \frac{4 C\|a(0)\|}{(t-\tau)^{1 / 2}}<\frac{1}{2}
$$

and also let

$$
C_{t_{0}}:=\frac{4 C\|a(0)\|}{\left(t-t_{0}\right)^{1 / 2}} \quad \text { and } \quad C_{T}:=\int_{0}^{T} \frac{C}{(T-\tau)^{1 / 2}}
$$

Then we have from (32) that

$$
\begin{aligned}
\left\|\mathcal{P}_{\eta} \tilde{y}(t)\right\| & \leq \frac{\alpha(\eta)}{\beta}\left(1+\eta^{2}\right)^{1 / 2}\|a(0)\|^{2}+\int_{t_{0}}^{t} 2 C_{t_{0}}\left\|\mathcal{P}_{\eta} \tilde{y}(\tau)\right\| d \tau+\epsilon C_{T} \\
& \leq \frac{\alpha(\eta)}{\beta}\left(1+\eta^{2}\right)^{1 / 2}\|a(0)\|^{2}+\int_{0}^{t} 2 C_{t_{0}}\left\|\mathcal{P}_{\eta} \tilde{y}(\tau)\right\| d \tau+\epsilon C_{T}
\end{aligned}
$$


for $0<t \leq T$. Application of Gronwall's inequality shows that

$$
\left\|\mathcal{P}_{\eta} \tilde{y}(t)\right\| \leq\left(\frac{\alpha(\eta)}{\beta}\left(1+\eta^{2}\right)^{1 / 2}\|a(0)\|^{2}+\epsilon C_{T}\right) e^{2 t C_{t_{0}}} \quad \text { for } \quad 0<t \leq T
$$

Note that $C_{T}$ and $C_{t_{0}}$ are independent of $\beta$. Thus for any $\tilde{\epsilon}>0$, there is $\eta_{0}$ and $\beta_{0}$ (depending on $\eta_{0}$ ) such that if $\eta>\eta_{0}$ and $|\beta|>\beta_{0}$, then $\left\|\mathcal{P}_{\eta} \tilde{y}\right\|<\tilde{\epsilon}$ for $0<t<T_{L}$. Clearly, we can also control $\left(I-\mathcal{P}_{\eta}\right) \tilde{y}$ with sufficiently large $\eta$ (independently of $\beta$ ), and $\mathcal{P}_{\eta} \tilde{B}^{0+}\left(\beta t, \mathcal{P}_{\eta} c, \mathcal{P}_{\eta} c\right)$ with a sufficiently large $\beta$ for fixed $\eta$. Therefore we can control $r(t)$ for sufficiently large $\eta$ and $\beta$, which completes the proof.

\section{Concluding remarks}

We have considered the time evolution of two-dimensional incompressible flow on a $\beta$ plane with the nonlinear terms being restricted to resonant wavenumbers. We have proved that the nonresonant nonlinear interactions are negligible when $\beta$ (the differential rotation rate) is sufficiently large. The resonant nonlinear interaction therefore dominates over the flow evolution, and the distribution of the resonant wavenumbers in wavenumber space (Fig.1) is quite anisotropic in wavenumber space. We note that the anisotropic energy spectral form of the $\beta$ plane turbulence has been discussed by [14].

It should be remarked here that our theorem applies to the flow field at $t \in\left[0, T_{L}\right]$, where $T_{L}$ is a local existence time of solution. It is commonly believed from large-scale numerical study in physics and earth and planetary sciences, that a smooth and unique solution to the governing equation exists in $t \in[0, \infty)$. Even when $T_{L}=\infty$, however, our proof is valid only in a finite interval of time although the time interval can taken be as long as desired.

Lastly, in many numerical works, a superviscosity $\nu_{p}(-\Delta)^{p} \zeta(p$ is a positive integer) has been employed instead of the normal viscosity $\nu \Delta \zeta$ in (1), in order to have a sufficiently wide inertial range, without the reduction of the enstrophy dissipation rate. We note that our main theorem also applies to the case of the superviscosity ${ }^{2}$.

Acknowledgements The first author is partially supported by the Japan Society for the Promotion of Science through the KAKENHI 20340018 and 22654014. The second author is partially supported by JST CREST.

$\overline{2 p>1} / 2$ is sufficient for our theorem to hold if $p$ is taken as a real number. 


\section{References}

[1] A. Babin, A. Mahalov and B. Nicolaenko, Regularity and integrability of $3 D$ Euler and Navier-Stokes equations for rotating fluids. Asymptot. Anal., 15 (1997), 103-150.

[2] A. Babin, A. Mahalov and B. Nicolaenko, Global regularity of the 3D Rotating Navier-Stokes Equations for resonant domains. Indiana University Mathematics Journal, 48 (1999), 1133-1176.

[3] J.Charney, The dynamics of long waves in a baroclinic westerly current. J.Meteorol. 4, 135, (1947).

[4] J.G.Charney, On the scale of atmospheric motions. Publ. Kosjones. Nors. Videnshap. Akac. Oslo, 17,3, (1948).

[5] J. Y. Chemin, B. Desjardins, I. Gallagher and E. Grenier, Mathematical geophysics. An introduction to rotating fluids and the Navier-Stokes equations. Oxford Lecture Series in Mathematics and its Applications, 32. The Clarendon Press, Oxford University Press, Oxford, (2006).

[6] Y. Giga, K. Inui, A. Mahalov and S. Matsui, Uniform local solvability for the Navier-Stokes equations with the Coriolis force. Methods Appl. Anal., 12 (2005), 381-393.

[7] Y. Giga, K. Inui and A. Mahalov and J. Saal, Uniform global solvability of the rotating Navier-Stokes equations for nondecaying initial data. Indiana University Mathematics Journal, 57 (2008), 2775-2791.

[8] Y. Giga, H. Jo, A. Mahalov and T. Yoneda, On time analyticity of the NavierStokes equations in a rotating frame with spatially almost periodic data. Physica D, 237 (2008), 1422-1428.

[9] S. Ibrahim and T. Yoneda, Long-time solvability of the Navier-StokesBoussinesq equations with almost periodic initial large data. submitted.

[10] E.Kartashova and V.S.L'vov, Cluster dynamics of planetary waves. Europhysics Letters, 83 (2008), 50012.

[11] J. Pedlosky, Geophysical Fluid Dynamics. 2nd edn, Springer, (1987).

[12] P. B. Rhines, Waves and turbulence on a beta plane. J. Fluid Mech., 69 (1975), 417-443.

[13] L.M.Smith and F.Waleffe, Transfer of energy to two-dimensional large scales in forced, rotating three-dimensional turbulence. Phys. Fluids, 11(1999)1608-1622, doi: $10.1063 / 1.870022$.

[14] G.K.Vallis and M.E.Maltrud, Generation of Mean Flows and Jets on a Beta Plane and over Topography. J. Phys. Oceangr., 23 (1993) 1346-1362. 
[15] T. Yoneda, Long-time solvability of the Navier-Stokes equations in a rotating frame with spatially almost periodic large data. Arch. Ration. Mech. Anal., 200 (2011), 225-237. 